\title{
Assessing Low-level Cognitive Processes of Word Recognition
}

\author{
Michael Holsworth \\ Kyoto Sangyo University
}

\begin{abstract}
A fundamental skill required for vocabulary development is word recognition ability. According to Perfetti (1985), word recognition ability relies on low-level cognitive processing skill to be automatic and efficient in order for cognitive resources to be allocated to high-level processes such as inferencing and schemata activation needed for reading comprehension. The low-level processes include orthographic knowledge, semantic knowledge, and phonological awareness. These low-level processes must be efficient, fluent, and automatic in second language readers in order for them to achieve the ultimate goal of reading comprehension. This article briefly describes the concept of word recognition, its relation to vocabulary, and three tests that were designed to measure the three components of word recognition (orthographic, semantic, and phonological knowledge) in a longitudinal study that investigated the effects of word recognition training on reading comprehension.
\end{abstract}

Key words: Reading, Word Recognition, Orthographic Knowledge, Semantic Knowledge, Phonological Awareness, Testing

\section{Introduction}

Reading is a fundamental aspect of communication and is a critical skill needed for successful L2 language learning. This skill, however, is difficult to develop for many learners when one considers the variety of different orthographic systems used around the world. This difference is easily seen between two orthographically different languages such as English and Japanese. It is vital to have a strong subset of reading skills that are automatic, such as word recognition, in order to develop a strong vocabulary for speaking and reading.

In order for an L2 learner to build his or her vocabulary and reading proficiency, the fundamental skill of word recognition is required. Richards and Schmidt (2002, p. 557) defined word recognition, a straightforward definition, as "higher level information is knowledge of permissible words as well as actual words of a language, while the lower level information is the actual phonetic input (or orthographic input in the case of written word recognition)" . This definition is rather narrow and focuses on the orthographic element of word recognition. Koda (1988, p. 135) defined word recognition as "the process of deriving both a phonological code (i.e., sound used in speech) and the meaning from graphemic representation." Koda (2004, p. 29) added to this definition of word recognition as 
"the processes of extracting lexical information from graphic displays of words" and relates only to a word's sounds and meaning. She further stated that decoding pertains to the extraction of phonological information.

In order for reading to be successful, several complex cognitive processes must occur rapidly, accurately, and automatically. These cognitive processes include low-level processes such as word recognition, semantic parsing, and semantic proposition encoding. In fact, according to Perfetti's Verbal Efficiency Theory (1985), reading comprehension is limited by a reader's low-level processing efficiency. When lower-level processes are automatic and efficient, a reader should be able to reallocate some of his or her cognitive resources to higher-level processes such as schemata activation, global knowledge application, and inferencing (Grabe, 2009; Perfetti, 1985). It is these high-level processes that lead to reading comprehension. It has been hypothesized that these higher-level processes rely upon lower-level processes to be efficient and automatic. In other words, highlevel processing used for reading comprehension relies on automatic low-level processes such as word recognition.

Perfetti and Hart (2001) defined word recognition as the interaction between three components: orthographic, phonological, and semantic processes. According to Cunningham, Perry, and Stanovich (2001), the visual recognition of word forms or orthographic knowledge is the first component and is one of the vital processing skills of word recognition. Orthographic processing includes identifying not only letters and letter groups but also word roots and morphological affixes. In addition, an understanding of how words are assembled using graphic representations is crucial to word recognition skill. The second component of word recognition is phonological processing and can be thought of as the awareness of the sound structures of a language (Stanovich, 1988). It is necessary to understand what sounds orthographic symbols of a language represent. Phonological awareness is known to be a key predictor of reading ability for both L1 and L2 English reading (Huang, Lin, \& Su, 2004; Johnson \& Tweedie, 2010; Jongejan, Verhoeven, \& Siegel, 2007; Swank \& Catts, 1994). This means that in order for L1 and L2 readers to process reading efficiently, they need to understand the graphophonemic mapping that occurs when decoding letters and words from a given language. It is seen as the precursor to semantic activation and is triggered by the onset of orthographic processing. For example, when a reader processes a word, the orthographic and phonological processes begin by processing the visual and sound cues. This leads to the third component of word recognition, semantic knowledge. A reader activates his or her semantic knowledge from his or her lexicon until the combination of orthographic, phonological, and semantic information finds the best match to the word being read if it is a word known to the reader, in other words, successful word recognition (Grabe, 2009). For L2 reading teachers, the logical goal should then be to foster the development of automatic word recognition skills by providing large amounts of comprehensible texts and training that targets word recognition skill development. A study by Burrows and Holsworth (2016) investigated this aspect of word recognition training and found that when students received training in all three components of word recognition, reading comprehension scores increased. This type of training seems to help learners 
move on from processing texts on a word-by-word basis. Teaching reading in the $\mathrm{L} 1$ and $\mathrm{L} 2$ requires knowledge of word recognition components in order to make sound pedagogical decisions to help learners improve their reading skill.

\section{Measurement of Word Recognition}

In order to have sufficient vocabulary in a second language to achieve reading comprehension, a learner must first develop and automatize word recognition skills. According to the Verbal Efficiency Theory (Perfetti, 1985), these skills are considered low-level cognitive processes and are easily automatizable. They include orthographic knowledge, semantic knowledge, and phonological awareness. These three components of word recognition are the key constructs to measure in order to accurately measure word recognition. To investigate this, a lexical decision test was used to measure orthographic knowledge, an antonym pairs test was selected to measure semantic knowledge, and a pronunciation speaking test was used to assess phonological awareness. The following is a description of each of these tests as they were used in a longitudinal study of the effects of word recognition training on reading comprehension.

\subsection{Lexical Decision Test}

The lexical decision test was designed to measure the orthographic knowledge of a reader. Participants are presented with a series of real English words and non-words. The original test was created by Matsuo (2017) and consists of 144 items (72 real English words and 72 non-words). Because reaction tests such as these can produce a cognitive test bias toward either positive or negative responses, and equal number of real-word items and non-word items were used (Jiang, 2012). By doing so, this reduces the possibility that a test taker would preemptively respond to an item based on his or her previous response. The real-word items were chosen from the British National Corpus with 18 items per level selected from the 1,000, $2,000,3,000$, and 4,000 level of frequency. These 18 items per frequency level were then equally divided into three subgroups, based on word length of four-, five-, and six-letter words. Finally, each word length subgroup contained two words representing a part of speech with two nouns, two verbs, and two adjectives. The non-words (or pseudowords) were created using the ARC Non-word Database (Rastle, Harrington, \& Coltheart, 2002). These non-words were based on English language spelling conventions in order to resemble real words and consist of legal bigrams and orthographically existing onsets. The non-words were also created to match the real-word items in word length.

This test was administered using the reaction time software called SuperLab (Version 5.0, 2014). Participants took the test individually using a standard laptop computer. Instructions appeared in the test taker's L1 (Japanese) on the screen followed by 14 practice items to acclimate the test taker to the test format. The 144 items were presented in random order for each participant. Prior to each item, visual primer image $(+++++)$ appeared on the screen for $1,000 \mathrm{~ms}$ so test takers were aware of the word location. After the test item appears, the 
test taker selected either the V-key for a real word or the N-key for a non-word. The responses were then recorded in millisecond reaction times. In L1 research, responses with $<200 \mathrm{~ms}$ are considered to be a preemptive guess, and a response of $>2,000 \mathrm{~ms}$ is considered to be a distracted response, and thus, they were considered outliers (Balota \& Chumbley, 1984). However, according to Jiang (2012), L2 reaction time boundaries need to be reconsidered due to the additional processing time required by participants. For this reason, responses with $200 \mathrm{~ms}$ or less were still considered preemptive guesses and were removed, and there was no maximum limit for response times.

The original test created by Matsuo consisted of one version only. However, the longitudinal study consisted of three test times, so two additional tests were created in order to have multiple measures and to avoid test bias. The original version had 72 target words, so with the addition of 12 anchor items present in the first test, the second and third tests contained 84 target words with corresponding non-word items. The anchor items allow for the measurement of the same items on all three tests and thus alleviate any potential issue with differences among the three tests. Table 1 shows the 12 anchor items present in the lexical decision test for all three test times.

\subsection{Antonym Pairs Test}

The antonym pairs test addresses the component of semantic knowledge and is categorized as a semantic classification task (Jiang, 2012). It has been used extensively as a semantic knowledge measure in word recognition research (Nation \& Snowling, 1998; Perfetti \& Zhang, 1995; Segalowitz \& de Almeida, 2002). The main goal of this test for test takers is to identify pairs of items as either antonyms or non-antonyms. The original version of this test was created by Matsuo (2014), and it consists of 72 antonym pairs and 72 non-antonym pairs. Examples of antonym pair items include life-death and come-go. Examples of non-antonym pairs

Table 1. Anchor Items - Lexical Decision Test

\begin{tabular}{lccc}
\hline Anchor item & Item counterpart & Part of speech & Frequency level \\
\hline Left & Meve & Noun & 1,000 \\
Laugh & Cromb & Verb & 1,000 \\
late & Pson & Adjective & 1,000 \\
Aunt & Kest & Noun & 2,000 \\
Smile & Phows & Verb & 2,000 \\
Empty & Skump & Adjective & 2,000 \\
Guest & Muilt & Noun & 3,000 \\
Shout & Telve & Verb & 3,000 \\
Angry & Orked & Adjective & 3,000 \\
Kilometer & Qweens & Noun & 4,000 \\
Kiss & Hosh & Verb & 4,000 \\
Ugly & Yoaf & Adjective & 4,000 \\
\hline
\end{tabular}

Vocabulary Learning and Instruction, 9 (2), 55-62. 
include plant-brother and fat-gray. In alignment with the lexical decision test, test items were selected to represent the first four 1,000 word frequency levels of the British National Corpus.

As with the original lexical decision test, the original antonym pairs test by Matsuo had only one version. Therefore, two new versions were created following the same criteria as the original test. The two new tests each contained 72 antonym pairs and 72 non-antonym pairs, with 18 pairs at each of the first four 1,000 word frequency levels. In following with the original test, the two new tests were controlled to the extent possible for part of speech and world length for the target words. The pair and non-pair items were also controlled to ensure that the primer word (first word shown) and the target word (second word shown) were at the same frequency level, but in some cases due to the limited choices for accurate antonym pairs, the difference between several items was no more than one frequency level. Once the three versions were created, they were combined and items were redistributed using random stratified assignment to ensure that each new version of test one, two, and three all had equal item representation from the original and new tests. Once again, the antonym pairs tests each contain the same 12 anchor items used in the lexical decision tests. Table 2 shows the anchor items used and their corresponding antonym or non-antonym pair.

Once again, the SuperLab software was used to measure participant reaction times in milliseconds. After reading the instructions, the next screen informed them they had 14 practice items before the test began. A visual primer appeared on the screen $(++++)$ for $1,000 \mathrm{~ms}$ to show the test taker where the words would appear. The primer $(++++)$ appears for $1,000 \mathrm{~ms}$ followed by the primer word for $1,000 \mathrm{~ms}$, which was then followed by the target word. Participants determined if the pair of words was an antonym pair or not. Participants pressed either the V-key to show they were antonym pairs or the N-key to show that it was a non-antonym pair. The reaction time was measured from the time the target word was shown until the student pressed either the V-key or N-key.

Table 2. Anchor Items - Antonym Pairs Test

\begin{tabular}{lllc}
\hline Anchor item & Antonym/non-antonym pair & Part of speech & Frequency level \\
\hline Left & Right & Noun & 1,000 \\
Laugh & Drive & Verb & 1,000 \\
Late & Early & Adjective & 1,000 \\
Aunt & Uncle & Noun & 2,000 \\
Smile & Cry & Verb & 2,000 \\
Empty & Occupied & Adjective & 2,000 \\
Guest & Manager & Noun & 3,000 \\
Shout & Leap & Verb & 3,000 \\
Angry & Final & Adjective & 3,000 \\
Kilometer & Earth & Noun & 4,000 \\
Kiss & Arrest & Verb & 4,000 \\
Ugly & Beautiful & Adjective & 4,000 \\
\hline
\end{tabular}

Vocabulary Learning and Instruction, 9 (2), 55-62. 


\subsection{Pronunciation Test}

The third test was a pronunciation test designed to measure participants' phonological awareness. The foundation for this test comes from the speaking section of the Wide Range Achievement Test (WRAT-4) by PAR (Wilkinson \& Robertson, 2006). The original WRAT-4 speaking section contains two tests with 55 items per test. Each test contains 55 items selected from the Education Development Laboratories (EDL) Core Vocabularies in Reading, Mathematics, Science, and Social Science (Taylor, Frackenpohl, \& White, 1989); and A Writing Vocabulary of Elementary Children (Hillerich, 1978). Each test begins with 15 warming up items (upper case letters of the English alphabet) to acquaint the test taker with the test format. The remaining items then move from common high-frequency words to less common low-frequency words. Test takers simply read the test items aloud, and they are judged to be either correct or incorrect. The original test was designed to be taken in a 1-to-1 format with a test taker and judge.

In order to have three equal versions of the pronunciation test for the longitudinal study, an additional new version was created. The original WRAT-4 test manual did not contain a description of the criteria for item selection, but contains only the source for the items. Therefore, the original two versions were analyzed for word length, phoneme count, syllable count, and frequency level on the British National Corpus. Once an additional set of items were selected from the same source as the original two tests, the three tests were then combined, and items were redistributed using randomly stratified allocation to create three new and equal pronunciation tests. Pilot data showed that each new test was not statistically significantly different from the other.

Test takers completed the pronunciation tests using a standard laptop computer and a headset. Test takers viewed the items using Microsoft PowerPoint and were instructed to say each item to the best of their ability and not be concerned about the word meaning. Time was not recorded for this test because accuracy was the main measure, not semantic or orthographic access speed (Jiang, 2012). The test results were then analyzed using five North American English speakers each with a high Japanese L2 speaking ability and over 10 years of post-secondary teaching experience in Japan. Each rater was coached to ensure they clearly understood the test and how to asses each speaker. Each interrater listened to their assigned audio files and determined if the speakers were correct or incorrect. Replies from test time one, two, and three were also randomly assigned so as to avoid rater bias on improvement. Individual accents were not considered a factor in the assessment; instead, each word was assessed as incorrect if it was misread by using grapheme-morpheme rules of English. For example, if a student added an additional voiced vowel to any part of a word, such as great pronounced as $g u$ reat-o, it was marked incorrect. Each interrater also used the Merriam-Webster Online Dictionary to access the syllable counts and audio samples to calibrate and clarify any item pronunciations (www.merriam-webster.com). The main research evaluated all audio files, and each interrater evaluated approximately 50 items, with an overlap of 10 items per interrater. This allowed for accurate Rasch assessment of interrater reliability of scoring. 


\section{Summary}

As an important part of reading, vocabulary knowledge relies upon strong word recognition skill. Understanding this foundational skill and its components provides greater insight into vocabulary development and reading skill growth. Through longitudinal research, measurement, and analysis of orthographic, semantic, and phonological knowledge, researchers add to the knowledge of vocabulary assessment and second language reading. In addition, L2 teachers will also be able to make better pedagogical choices to help their students achieve their learning and reading goals, for example, by including training tasks designed to move readers from a word-by-word low-level cognitive processing to a more highlevel cognitive processing of texts that better facilitate reading comprehension.

\section{References}

Balota, D., \& Chumbley, J. (1984). Are lexical decisions a good measure of lexical access? The role of word frequency in the neglected decision stage. Journal of Experimental Psychology, 10(3), 340-357. doi:10.1037/0096-1523.10.3.340

Burrows, L., \& Holsworth, M. (2016). The effects of explicit word recognition training on Japanese EFL learners. The Reading Matrix: An International Online Journal, 16(2), 81-97.

Cunningham, A. Perry, K., \& Stanovich, K. (2001). Converging evidence for the concept of orthographic processing. Reading and Writing: An Interdisciplinary Journal, 14(5), 549-568. doi:10.1023/A:1011100226798

Grabe, W. (2009). Reading in a second language: Moving from theory to practice. New York, NY: Cambridge University Press.

Hillerich, R. (1978) A writing vocabulary of elementary children. Springfield, IL: C. C. Thomas.

Huang, C., Lin, S., \& Su, B. (2004). The effects of phonological awareness training on technological university students' phonics and vocabulary knowledge. Journal of National Taipei Teachers College, 17(1), 59-90. Retrieved from http:// readopac1.ncl.edu.tw/nclJournal/search/detail.jsp?sysId=0004801243\&dt$\mathrm{dId}=000040 \&$ search_type $=$ detail\&la $=\mathrm{ch}$ 國家圖書館

Jiang, N. (2012). Conducting reaction time research in second language studies. New York, NY: Routledge.

Johnson, R., \& Tweedie, G. (2010). Could phonemic awareness instruction be (part of) the answer for young EFL learners? A report on the early literacy project in Malaysia. TESLO Quarterly, 44(4), 822-829. doi:10.5054/tq.2010.238131

Jongejan, W., Verhoeven, L., \& Siegel, L. (2007). Predictors of reading and spelling abilities in first- and second-language learners. Journal of Educational Psychology [H.W. Wilson - EDUC], 99(4), 835-851. doi:10.1037/0022-0663. 99.4.835

Koda, K. (1988). Cognitive process in second language reading: Transfer of L1 reading skills and strategies. Second Language Research, 4(2), 133-155. doi:10.1177/026765838800400203 
Koda, K., (2004). Insights into second language reading: A cross linguistic approach. Cambridge, UK: Cambridge University Press.

Matsuo, T. (2017). The Roles of Lexical Size, Depth, and Automaticity of Word Recognition on Reading Comprehension (Unpublished doctoral dissertation). Temple University, Tokyo, Japan

Nation, K., \& Snowling, M. (1998). Semantic processing and the development of word-recognition skills: Evidence from children with reading comprehension difficulties. Journal of Memory and Language, 39, 85-101. doi:10.1006/ jmla.1998.2564

Perfetti, C. A. (1985). Reading ability. New York, NY: Oxford Press.

Perfetti, C. A., \& Hart, L. (2001). The lexical bases of comprehension skill. In D. Gorfien (Ed.), On the consequences of meaning selection (pp. 67-86). Washington, DC: American Psychological Association.

Perfetti, C. A., \& Zhang, S. (1995). Very early phonological activation in Chinese reading. Journal of Experimental Psychology, 21(1), 24-33. doi:10.1037/ 0278-7393.21.1.24

Rastle, K., Harrington, J., \& Coltheart, M. (2002). 358,534 nonwords: The ARC Nonword Database. Quarterly Journal of Experimental Psychology, 55A, 1339-1362. doi:10.1080/02724980244000099

Richards, J. C., \& Schmidt, R. (2002). Longman dictionary of language teaching \& applied linguistics (3rd ed.). Harlow, UK: Pearson Education Limited.

Segalowitz, N., \& de Almeida, R. (2002). Conceptual representation of verbs in bilinguals: Semantic field effects and a second-language performance paradox. Brain and Language, 81(1), 517-531. doi:10.1006/brln.2001.2544

Stanovich, K. E. (1988). The right and wrong places to look for the cognitive locus of reading disability. Annals of Dyslexia, 38(1), 154-177. doi:10.1007/ BF02648254

SuperLab (Version 5) [Computer software]. (2014). San Pedro, CA: Cedrus Corporation. Retrieved from http://www.superlab.com/v5/

Swank, L., \& Catts, H. (1994). Phonological awareness and written word decoding. Language, Speech, and Hearing Services in Schools, 25(1), 9-14. Retrieved from http://vv4kg5gr5v.search.serialssolutions.com.libproxy.temple.edu/

Taylor, S. E., Frackenpohl, H., \& White, C. E. (1989). A revised core vocabulary. $E D L$, EDL core vocabularies in reading, mathematics, science, and social studies. Columbia, SC: Educational Developmental Laboratories.

Wilkinson, G., \& Robertson, G. (2006). WRAT4 Wide range achievement test professional manual. Lutz, FL: PAR. 